Case Report

\title{
Chromosome 20p Partial De Novo Duplication Identified in a Female Paediatric Patient with Characteristic Facial Dysmorphism and Behavioural Anomalies
}

\author{
Shahzaib Khattak, ${ }^{1,2}$ Meryam Jan, ${ }^{1}$ Sara Warsi, ${ }^{1,2}$ and Sohail Khattak ${ }^{1}{ }^{1}$ \\ ${ }^{1}$ The Kids Clinic, 300 Rossland Rd E, Suite 301, Ajax, ON L1Z 0M1, Canada \\ ${ }^{2}$ McMaster University, 1280 Main St W, Hamilton, ON L8S 4L8, Canada \\ Correspondence should be addressed to Sohail Khattak; skhattak@kidsclinic.ca
}

Received 23 August 2019; Revised 2 December 2019; Accepted 29 February 2020; Published 11 July 2020

Academic Editor: Christos Yapijakis

Copyright (c) 2020 Shahzaib Khattak et al. This is an open access article distributed under the Creative Commons Attribution License, which permits unrestricted use, distribution, and reproduction in any medium, provided the original work is properly cited.

Copy number variations (CNVs) involving the JAG1 gene are rare and infrequently reported in the scientific literature. Recently, a generally healthy young patient presenting with a history of behavioural concerns was referred to us. Herein, we discuss the patient, a 7 -year-old female possessing a $0.797 \mathrm{Mb}$ microduplication within the short arm of chromosome 20 at band 12.2. The patient generates considerable curiosity due to the rarity of her case, which includes a de novo partial duplication involving the JAG1 gene. The patient exhibits a wide range of symptoms including facial dysmorphism (dolichocephaly, round face, tented philtrum, anteverted nares, and micrognathia), clinodactyly, and an inborn congenital heart defect. She presented with behavioural concerns including ADHD-I, SPD, motor clumsiness, and poor self-regulation. Deletions in JAG1 are often linked to Alagille Syndrome; however, complete duplications have not been specifically identified as disease-causing. JAG1 mutations are reported alongside various clinical features including facial dysmorphology, heart defects, vertebral abnormalities, and ocular dysmorphic features (strabismus, epicanthal folds, and slanted palpebral fissures). This particular microduplication is rare, and thus, limited data exist regarding its significance. To our knowledge, most reported duplications are larger than $0.797 \mathrm{Mb}$. This may define a critical region causing phenotypical changes in some patient cases.

\section{Introduction}

Information concerning de novo genetic aberrations on the short arm of chromosome 20 is absent from the current scientific literature. JAG1 mutations are primarily associated with Alagille syndrome [1-5] caused by microdeletions. Also, variations in JAG1 are associated with multiple types of cancer including breast cancer and adrenocortical carcinoma $[6,7]$. Pure trisomy $20 \mathrm{p}$ is infrequently reported $[8,9]$. Clinical characteristics of mutated $20 \mathrm{p}$ with regard to JAG1 are seen in Alagille syndrome [6] and are correlated with deregulation of NOTCH signaling. JAG1 acts as a ligand in the NOTCH signaling pathway, which plays an important role in cell signaling and cell membrane protein transportation. NOTCH is a key component of embryogenesis, organogenesis, and cell fate specification [10]. Since JAG1 acts as a ligand in the NOTCH signaling pathway, JAG1 gene deletions would downregulate the Notch signaling [11]. JAG1 deletions risk interrupting the NOTCH signaling pathway. 20p duplications are less common than deletions and typically show a wider range of phenotypic traits. Variability is based on the size of the duplicated segment. Symptoms include, but are not limited to, developmental delay, round facial structure, prominent cheeks, and ocular abnormalities (slanted palpebral fissures or strabismus) $[8,9,11,12]$.

Our focus of discussion is a rare de novo $20 \mathrm{p}$ microduplication at band 12.2 seen in a young female patient with delayed speech and intellectual disability (ID) (based on DSM-5 criteria, our patient suffers from intellectual 
disability (intellectual development disorder) severity level: mild DSM-5 Code F70). ID is a disorder involving intellectual and adaptive functioning deficits with onset during the developmental period. Intellectual functions involve deficiencies in reasoning, problem solving, abstract thinking, etc. with IQ scores ranging between 65 and 70 . Adaptive functions involve failing to adhere to developmental and social cultural standards, poor motor skills, ADHD (predominantly inattentive presentation) (ADHD-I), sensory processing disorder, characteristic dysmorphia, and congenital heart defects.

\section{Case Presentation}

In February 2016, the patient was referred to our clinic for a routine evaluation of behavioural issues. Her age, at this time, was 7 years and 3 months. The main concerns regarding the patient were behavioural issues such as an inability to focus, frequent temper tantrums, sensitivity to loud noises, and foul language. She was a generally healthy child with no seizures, medications, or recent hospitalizations. The patient was conceived via intrauterine insemination (IUI) as a twin pregnancy between nonconsanguineous Caucasian parents. The mother underwent amniocentesis during pregnancy. Developmental history showed prenatal complications involving a blood clot at 7 months of gestation. For the remainder of the pregnancy, the mother was put on Fragmin (dalteparin). Fetal karyotypes were reportedly normal. At 37 weeks of gestation, the mother was induced and she had a vaginal delivery. Further complications arose during delivery when it was noticed that the arm was positioned above the head, requiring vacuum extraction. In the postpartum period, the patient's haematology panel showed hypoglycaemia. She was kept in a neonatal intensive care unit (NICU) for 36 hours after delivery. The blood sugar recovered in the first 12 hours. The patient was observed for an additional 24 hours and was discharged thereafter. Her birth weight was recorded at 5 pounds, 10 ounces. Apgar scores were normal. At birth, an abdominal and renal ultrasound showed stasis nephropathy. Subsequently, a voiding cystourethrogram (VCUG) was done and it showed normal renal structure with no reflux.

The patient was noted to have facial dysmorphic features and a heart murmur. She went through extensive investigation and was diagnosed with atrial septal defect (ASD) (secundum), which was subsequently repaired due to a substantial left to right shunt and right ventricular (RV) dilation at the age of 4 . She had a persistent minor ventricular conduction delay (crochetage) in the right precordial leads due to ASD (Figure 1). The patient was noted to have facial dysmorphic features including anteverted nares, short nose, tented philtrum, round face, micrognathia, epicanthic folds, unilateral ptosis, and clinodactyly (Table 1). Strabismus and unilateral congenital ptosis were surgically corrected. Additionally, the patient had myringotomy tube insertions twice. Her head circumference in 2016 at age 8 was measured to be $53.1 \mathrm{~cm}\left(90^{\text {th }}\right.$ percentile). Her height was measured to be $115.1 \mathrm{~cm}$ ( $5^{\text {th }}$ percentile), and her weight was $23.3 \mathrm{~kg}\left(50^{\text {th }}\right.$ percentile). The patient's facial dysmorphic features and ASD resulted in clinical genetic consultation in 2011. Chromosomal karyotyping of peripheral blood was performed using standard protocols. Fluorescence in situ hybridization (FISH) was engineered using a BAC clone (RP11-103J8) which maps to chromosome 20, nucleotide position 10,840,138-10,994,545. Parental karyotypes were normal. The proband karyotype displayed gain at the $20 \mathrm{p}$ site, specifically registered as 46, XX, dup (20) (p12.2). The de novo occurrence of the microduplication was confirmed by FISH analysis that showed normal parental karyotypes. In order to determine the presence of any complex rearrangement, a genomic microarray was carried out to measure the expression levels of genomic sequences and CNVs using the genomic microarray platform CytoScan HD SNP Array (Affymetrix), genome build: NCBI 37/hg 19 (2009), and analysis software: ChAS (Affymetrix). Genomic microarray analysis showed a $0.797 \mathrm{Mb}$ duplication in chromosome region 20p12.2 that involves 6 RefSeq (Reference Sequence) genes: SLX4IP, MIR6870, LOC101929395, LOC101929413, LOC339593, and one OMIM Morbid Map gene: JAG1 (UCSC Genome Browser hg19). The distal breakpoint lies within the SLX4IP gene, resulting in partial gene duplication (Figure 2). The clinical significance of the $J A G 1$ duplication was indicated as unknown in the report because the literature regarding this group of CNVs was considered insufficient.

Neuroimaging was performed due to presentation of developmental delay. MRI was performed using multiplanar, multisequence imaging of the brain with axial T1, MP, RAGE and sagittal reformats, axial T2, axial proton density, diffusion imaging, coronal FLAIR, and axial T1 postgadolinium images in 2011. Results indicated dolichocephaly, with mild general volume loss. No focal intracranial abnormalities were detected.

Spinal MRI examinations were performed due to presentation of developmental delay. The technique used was sagittal T2 proton density, FIR, and T1 Angiary images of the cervical and thoracic spine with axial T2 images. Results indicated that the spine was visualized to the level of the L1 vertebral body. The cerebellar tonsils were in appropriate position. The cord was normal with normal cord signal and no cord compression. The tip of the conus was not seen, but it appeared to end just below L1. No segmentation or fusion anomalies were identified. Vertebral body heights were maintained throughout with a normal marrow signal.

The patient experienced developmental delays, where milestones were not met appropriately. She sat without support at 9 months and did not begin walking until 24 months. The patient's fine and gross motor skills, expressive and receptive language, and social skills were also delayed. The patient had difficulty forming sentences and effectively communicating with others. She struggled with social skills such as cooperatively engaging with other children and preferred parallel play. She experienced frequent mood swings and had an inability to appropriately express her feelings. Examination of the patient's family history revealed maternal anxiety and a paternal learning disability as well as a thyroid disorder. The patient's maternal grandfather and paternal grandparents were diagnosed with diabetes 


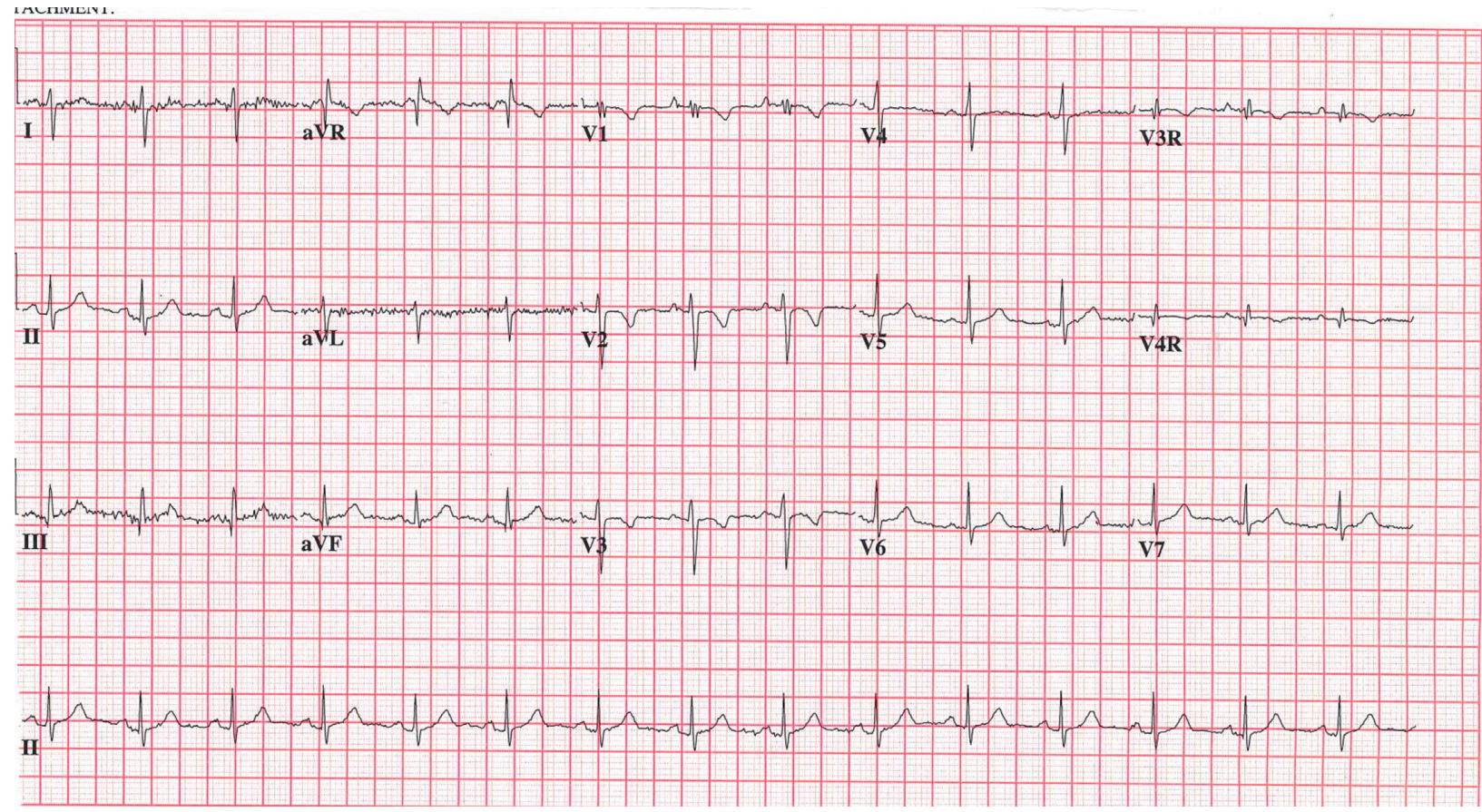

Figure 1: Patient's abnormal EKG ventricular conduction delay (crochetage).

mellitus. Both paternal grandparents have high blood pressure and heart disease. Based on the patient's extensive family history, assessment of her liver enzymes, triglycerides, sugars, etc. was recommended. The patient's fraternal twin brother had esotropia but otherwise is healthy.

The patient's most recent assessment at 7 years and 8 months of age indicates a continuation of social skills difficulties, poor emotional regulation, and difficulty maintaining friendships/relationships with peers and the sibling. She experiences unwarranted levels of worry and anxiety and sensory sensitivity to loud noises. The patient is currently diagnosed with sensory processing disorder (SPD), mild intellectual disorder (MID), and attention deficit hyperactivity disorder. Improvements in speech and language were observed after completion of speech therapy. Her most recently recorded cardiopulmonary assessments and haematology panels are all normal.

To the best of our knowledge, the patient is the first female patient reported to have a de novo microduplication within the JAG1 gene in conjunction with SPD, ID, and ADHD-I. Information on the clinical significance of the patient's chromosomal anomaly is currently unknown as there is a paucity of the published literature on JAG1 mutations.

\section{Discussion}

We present the case of a patient, a 7-year-old female, with a rare case involving a de novo partial duplication in the 20p12.2 region containing the JAG1 gene, confirmed by genomic hybridization and FISH. She did not have any other chromosomal abnormalities. The patient presented with intellectual disability, developmental delay, attention deficit hyperactivity disorder (predominantly inattentive presentation), and a sensory processing disorder. Review of medical records indicates that she had a repair of an atrial septal defect at 4 years of age. The patient exhibited classic clinical dysmorphic features of de novo partial 20p trisomy such as strabismus (surgically corrected), esotropia (surgically corrected), tented philtrum, and ptosis.

A microarray showed duplication to be within the short arm of chromosome $20(10,524,474-11,322,239)$, as confirmed by FISH. JAG1 is located in this region encoding a ligand in the NOTCH1 signaling pathway. Expression of the ligand encoded by the JAG1 gene plays a role in embryogenesis of the cardiovascular and renal systems [6].

Although microdeletion of JAG1 is known to be syndromic, duplication of $J A G 1$ - as seen in our patient-has not yet been identified as a risk factor for any particular disease. Pathogenic duplications of this region have been previously reported in the ISCA and Decipher databases; however, phenotypic information is limited, and previous cases had larger duplications than those detected in our patient $(0.797 \mathrm{Mb})$.

A review of the ISCA database for the patient's duplicated region of chromosome 20 displayed 3 reported losses and 11 gains with multiple size variants and phenotypes. Of the 11 gains reported, the variant size ranged from $0.49 \mathrm{Mb}$ to $63 \mathrm{Mb}$. The phenotypes observed for gains in this region include developmental delay and heart abnormalities which were evident in the patient. Additional phenotypes reported in this region are microcephaly, laryngomalacia, hypotonia, aganglionic megacolon, and hydronephrosis.

Microdeletions of JAG1 have been reported to contribute to features of Alagille Syndrome [1-5], which is a congenital disorder that clinically manifests in early childhood or infancy. One of the prominent features of the 
TABLE 1: Comparison of the patient's phenotypic characteristics versus published accounts of dup 20p/trisomy 20p.

\begin{tabular}{|c|c|c|c|c|c|}
\hline Phenotypic characteristics & Our patient & Grammatico et al. [8]* & Bartolini et al. [9] & Trachoo et al. [12] & Chaabouni et al. [13] \\
\hline Right congenital ptosis & + & - & + & - & - \\
\hline Epicanthic folds & + & - & + & + & + \\
\hline Upslanting palpebral fissures & + & - & + & + & - \\
\hline Strabismus & + & + & + & - & - \\
\hline Esotropia & + & - & - & - & - \\
\hline Round face & + & + & + & + & + \\
\hline Philtrum abnormalities & + & + & + & - & + \\
\hline Coarse hair & - & + & + & - & + \\
\hline Short neck & + & - & + & + & + \\
\hline Abnormal ears & + & + & + & - & + \\
\hline Nasal abnormalities & + & + & + & + & + \\
\hline Anteverted nares & + & - & + & - & + \\
\hline Palate (arched or cleft) & - & + & - & - & + \\
\hline Micrognathia & + & - & + & - & + \\
\hline Midface hypoplasia & - & - & - & + & - \\
\hline Hypertelorism & - & + & - & - & - \\
\hline Hearing deficit & - & - & - & + & - \\
\hline Thin upper lip & - & - & - & - & + \\
\hline Digital anomalies & + & + & + & - & + \\
\hline Macrocephaly & - & - & + & + & - \\
\hline Dolichocephaly & + & - & - & - & - \\
\hline Plagiocephaly & - & + & - & - & + \\
\hline Intellectual disability & + & + & + & + & + \\
\hline $\begin{array}{l}\text { Attention deficit hyperactivity } \\
\text { disorder }\end{array}$ & + & - & - & - & - \\
\hline Sensory processing disorder & + & - & - & - & - \\
\hline Speech delay & + & + & - & + & + \\
\hline Poor motor coordination & + & + & + & + & + \\
\hline Developmental delay & + & + & + & + & + \\
\hline Other behavioural issues & + & + & + & + & + \\
\hline Vertebral anomalies & - & + & - & - & + \\
\hline Renal anomalies & - & + & - & + & + \\
\hline Cardiac malformations & + & + & - & - & + \\
\hline Genital anomalies & - & + & - & - & + \\
\hline Skeletal anomalies & + & + & - & - & + \\
\hline Dental abnormalities & + & - & - & - & + \\
\hline
\end{tabular}

${ }^{*}$ Grammatico et al. is a case series involving two patients. Adapted from Grammatico et al. [8], Bartolini et al. [9], Trachoo et al. [12], and Chaabouni et al. [13].

syndrome is liver damage resulting from a lack of interlobular biliary ducts [5]. Pulmonic stenosis, ventricular septal defect (VSD), ASD, and tetralogy of Fallot (TOF) are also common cardiovascular abnormalities of patients with Alagille Syndrome [5]. Missense mutations in JAG1 resulting in VSD, ASD, and TOF have also been reported in patients that did not have Alagille Syndrome [14, 15]. In situ hybridization studies have previously demonstrated the importance of JAG1 expression in the vascular structures of the heart [16], with abnormalities caused by either a dysfunction of the NOTCH pathway ligand JAG1, or potentially confounding genetic modifiers beyond the detectable JAG1 mutation. Our patient presented with a partial duplication in JAG1 and had an ASD that was previously repaired.

There have been very few reported cases of patients with neuropsychiatric disorders and duplications within the $20 \mathrm{p}$ chromosome [17]. In 2004, a case series on genomic imbalances and intellectual disabilities included a patient who presented with a de novo duplication within 20p12.2 containing the JAG1 gene and suffered from mental retardation, behavioural aggression, and schizophrenia. The patient died at the age of 60 due to multiple myeloma after being institutionalized for over 40 years [18]. Our patient also displayed poor self-regulation and mild intellectual disability; however, given her age, it is uncertain whether comorbid diseases will manifest in adulthood. Chaabouni et al. [13] described a 5-year-old patient with de novo trisomy 20p of paternal origin of nearly the entire short arm of chromosome 20, with similar craniofacial dysmorphologies and intellectual disability as observed in the patient, including a round face with prominent cheeks and slanted palpebral fissures. Their patient also presented with hexadactyly, brachydactyly, and camptodactyly similar to our patient who presented with clinodactyly. Chaabouni's patient and our patient both had normal Apgar scores, developmental delay, moderate motor activity, and speech delay [13]. Other patient reports involving $20 \mathrm{p}$ duplications consistently report some of the characteristics seen in our patient (Table 1) including round face with prominent cheeks, speech delay, moderate intellectual disability, slanted palpebral fissures, and strabismus $[8,9,12]$. 


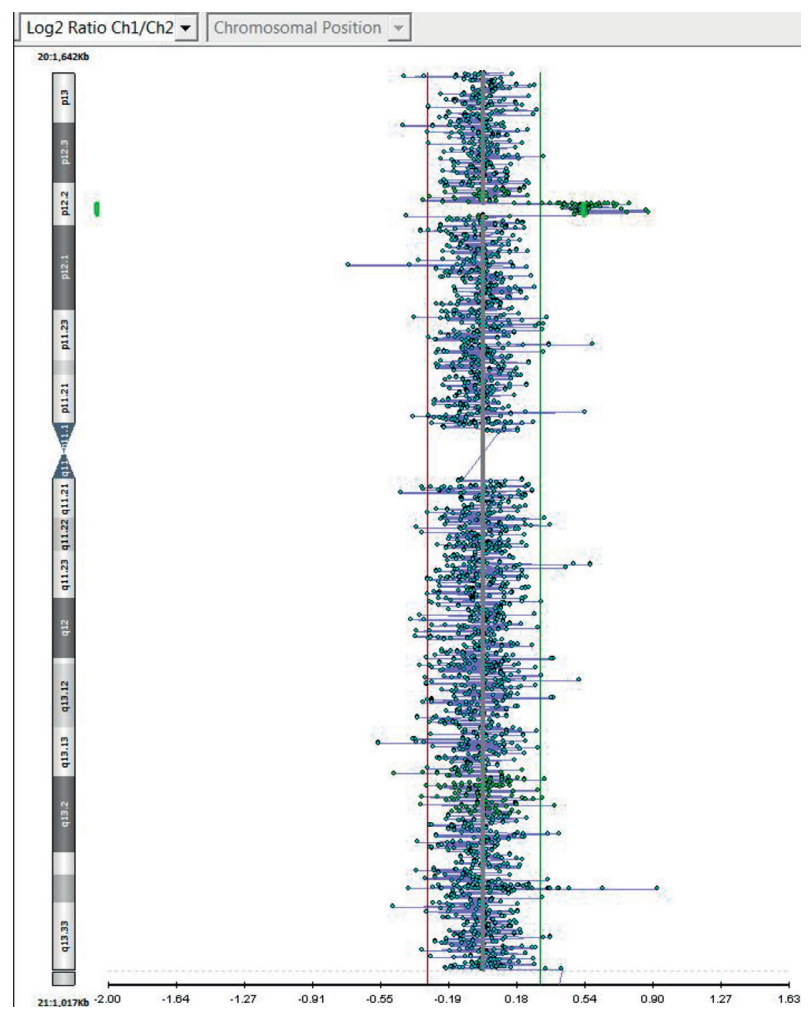

FIGURE 2: Detail of the array CGH showing duplication of chromosome $20(0.797 \mathrm{Mb})$ in the patient. Chromosomal view showing a deviation of baseline ( $\log 2$ ratio of -2$)$. Image obtained from Credit Valley Hospital.

Upon comparing the patient to other cases we found in the literature, we observed some phenotypic features that were common to all cases and some features shared by only some (Table 1). All reported cases, including that of our patient, had the following phenotypic traits in common: round face, nasal abnormalities, neurodevelopmental delay, intellectual disability, and other behavioural issues (Table 1). In addition, most patients in the aforementioned cases shared specific facial and ocular dysmorphic features (epicanthic folds, upslanting palpebral fissures, abnormal ears, philtrum abnormalities, micrognathia, strabismus, and anteverted nares) (Table 1). Our patient and the other patients described in Table 1, displayed evidence of skull abnormalities and short necks. Finally, most patient cases involved cardiopulmonary anomalies, which in our patient's case involved a surgically repaired large ASD (Table 1). This finding is especially interesting, as other researchers have identified similar mutations in nearby genomic regions that have generated other significant cardiopulmonary syndromes. In 2013, a case report described a female patient with an approximate $770 \mathrm{~Kb}$ duplication in the 20p12.3 region of the genome [19]. This patient also had Wolff-Parkinson-White syndrome, which is a congenital heart disorder that can result in episodic tachycardia [19]. It is important to note that this duplication is very similar in size and in close proximity to the $0.797 \mathrm{Mb}$ duplication found in the 20p12.2 region of our patient's genome. Another case report in 2013 discussed a patient with a $7.8 \mathrm{Mb}$ duplication in the $20 \mathrm{p} 12.2-11.23$ region of the genome [20]. This patient also presented with congenital heart disease (ASD), which was also documented in our patient [20]. Comparing our patient to the cases we have explored in the literature, we hypothesize that the $20 \mathrm{p} 12.2$ portion of the genome may serve as a critical region that when duplicated, results in the cardiac anomalies and other defects seen in our patient and other cases of chromosome 20p duplication discussed in this report.

The aim of this case report is to contribute to the current understanding of patients with duplications within the short arm of chromosome 20, as phenotypic information on these patients is not currently well reported. Our patient presented with a de novo partial duplication within the DNA region containing the JAG1 gene. As per the current literature, reported cases share common clinical features with few differences. We include an extensive genetic workup and clinical and behavioural assessments in order to assist clinicians when presented with these patients in their practices, to improve the care and management of children presenting with such genetic syndromes.

\section{Conflicts of Interest}

The authors declare that there are no conflicts of interest regarding the publication of this article.

\section{References}

[1] B. M. Kamath, B. D. Thiel, X. Gai et al., "SNP array mapping of chromosome 20p deletions: genotypes, phenotypes, and copy number variation," Human Mutation, vol. 30, no. 3, pp. 371-378, 2009.

[2] E. Legius, J.-P. Fryns, B. Eyskens et al., "Alagille syndrome (arteriohepatic dysplasia) and $\operatorname{del}(20)(\mathrm{p} 11.2)$," American Journal of Medical Genetics, vol. 35, no. 4, pp. 532-535, 1990.

[3] S. Moutton, C. Rooryck, J. Toutain et al., "Dysmorphic features in subtelomeric 20p13 deletion excluding JAG1: a recognizable microdeletion phenotype?" European Journal of Medical Genetics, vol. 55, no. 2, pp. 151-155, 2012.

[4] F. Zhang, J.-F. Deleuze, A. Aurias et al., "Interstitial deletion of the short arm of chromosome 20 in arteriohepatic dysplasia (Alagille syndrome)," The Journal of Pediatrics, vol. 116, no. 1, pp. 73-77, 1990.

[5] P. D. Turnpenny and S. Ellard, "Alagille syndrome: pathogenesis, diagnosis and management," European Journal of Human Genetics, vol. 20, no. 3, pp. 251-257, 2012.

[6] C. M. Grochowski, K. M. Loomes, and N. B. Spinner, "Jagged1 (JAG1): structure, expression, and disease associations," Gene, vol. 576, no. 1, pp. 381-384, 2016.

[7] M. Reedijk, S. Odorcic, L. Chang et al., "High-level coexpression of JAG1 and NOTCH1 is observed in human breast cancer and is associated with poor overall survival," Cancer Research, vol. 65, no. 18, pp. 8530-8537, 2005.

[8] P. Grammatico, F. Cupilari, C. D. Rosa, M. Falcolini, and G. D. Porto, " $20 \mathrm{p}$ duplication as a result of parental translocation: familial case report and a contribution to the clinical delineation of the syndrome," Clinical Genetics, vol. 41, no. 6 , pp. 285-289, 1992.

[9] L. Bartolini, S. Sartori, E. Lenzini et al., "De novo trisomy 20p characterized by array comparative genomic hybridization: report of a novel case and review of the literature," Gene, vol. 524, no. 2, pp. 368-372, 2013. 
[10] K. Shimizu, S. Chiba, T. Saito, K. Kumano, and H. Hirai, "Physical interaction of Delta1, Jagged1, and Jagged2 with Notch1 and Notch3 receptors," Biochemical and Biophysical Research Communications, vol. 276, no. 1, pp. 385-389, 2000.

[11] Johns Hopkins University, Online Mendelian Inheritance in Man, $O M I M^{\circledR}$, Johns Hopkins University, Baltimore, MD, USA, 2018, https://www.omim.org/entry/601920.

[12] O. Trachoo, M. Assanatham, N. Jinawath, and A. Nongnuch, "Chromosome 20p inverted duplication deletion identified in a Thai female adult with mental retardation, obesity, chronic kidney disease and characteristic facial features," European Journal of Medical Genetics, vol. 56, no. 6, pp. 319-324, 2013.

[13] M. Chaabouni, C. Turleau, L. Karboul et al., "De novo trisomy 20 p of paternal origin," American Journal of Medical Genetics Part A, vol. 143A, no. 10, pp. 1100-1103, 2007.

[14] R. C. Bauer, A. O. Laney, R. Smith et al., "Jagged1 (JAG1) mutations in patients with tetralogy of Fallot or pulmonic stenosis," Human Mutation, vol. 31, no. 5, pp. 594-601, 2010.

[15] Z. A. Eldadah, A. Hamosh, N. J. Biery et al., "Familial tetralogy of fallot caused by mutation in the jagged1 gene," Human Molecular Genetics, vol. 10, no. 2, pp. 163-169, 2001.

[16] K. M. Loomes, L. A. Underkoffler, J. Morabito et al., "The expression of Jagged1 in the developing mammalian heart correlates with cardiovascular disease in Alagille syndrome," Human Molecular Genetics, vol. 8, no. 13, pp. 2443-2449, 1999.

[17] G. S. Baroff and J. G. Olley, Mental Retardation: Nature, Cause, and Management, Routledge, Abingdon, UK, 2014.

[18] M. Kriek, S. J. White, M. C. Bouma et al., "Genomic imbalances in mental retardation," Journal of Medical Genetics, vol. 41, no. 4, pp. 249-255, 2004.

[19] K. I. Mills, J. Anderson, P. T. Levy et al., "Duplication of 20p12.3 associated with familial Wolff-Parkinson-White syndrome," American Journal of Medical Genetics Part A, vol. 161, no. 1, pp. 137-144, 2013.

[20] J. R. Batanian, S. R. Braddock, K. Christensen, and A. P. Knutsen, "Combined immunodeficiency in a 3-year-old boy with $16 \mathrm{p} 11.2$ and 20p12.2-11.2 chromosomal duplications," American Journal of Medical Genetics Part A, vol. 164, no. 2, pp. 535-541, 2014. 